\title{
INFANCIA: VULNERABILIDAD Y RESILIENCIA ${ }^{1}$
}

Childhood: vulnerability and resilience

Infância: vulnerabilidade e resiliencia

\section{Antonio Coronado Hijón \\ Universidad de Sevilla, España. Fono: +34 955420714. Correo electrónico: acoronado1@us.es}

\begin{abstract}
Resumen
Desde el Fondo Internacional de Emergencia de las Naciones Unidas para la Infancia (UNICEF), se viene haciendo llamamientos para la innovación en equidad e igualdad de oportunidades para la infancia.

La promoción de la resiliencia académica es una prometedora línea de innovación e investigación educativa, enmarcada en el ámbito de la educación emocional, que ofrece apoyos y garantías para superar dificultades y sobreponerse a ellas de manera exitosa.

Desde los ámbitos de actuación más relevantes de la educación de la resiliencia (el sociocomunitario y el escolar), la comunidad tiene un rol relevante desde un enfoque sistémico de la intervención.

Palabras clave: educación emocional, resiliencia académica, sociocomunitario.
\end{abstract}

\begin{abstract}
From the United Nations International Children's Emergency Fund (UNICEF), calls for innovation in equity and equal opportunities for children are being made.

The promotion of academic resilience is a promising line of innovation and educational research, framed in the field of emotional education, offering support and guarantees to overcome difficulties and overcome them successfully.

From the most relevant intervention areas of resilience education, the socio-community and the school, the community has a relevant role from a systemic approach to intervention.
\end{abstract}

\footnotetext{
${ }^{1}$ Ponencia invitada en el Congreso Internacional e Interuniversitario contra la pobreza infantil en el mundo.
} 
Keywords: emotional education, academic resilience, socio-community.

\section{Resumo}

A partir do Fundo de Emergência Internacional do Fundo das Nações Unidas para a Infância (UNICEF), que tem vindo a fazer chamadas para a inovação no capital próprio e igualdade de oportunidades para as crianças.

Promover a resiliência acadêmica é uma promissora linha de inovação educacional e de pesquisa, enquadrado no campo da educação emocional, oferecendo apoio e garantias para superar as dificuldades e superá-los com sucesso.

Desde as mais importantes áreas de intervenção resiliência educação, comunidade social e da escola, a comunidade tem um papel significativo a partir de uma abordagem sistémica à intervenção.

Palavras-chave: educação emocional, superação académica, comunidade social.

\section{Estado y necesidades de la Infancia.}

El United Nations International Children's Emergency Fund (UNICEF), en español (Fondo Internacional de Emergencia de las Naciones Unidas para la Infancia), elabora informes anuales sobre el estado mundial de la infancia, donde examina los factores más relevantes que afectan coyuntural y estructuralmente a la niñez alrededor del mundo.

En el año en que el mundo conmemoró los 25 años de la Convención sobre los Derechos del Niño, el informe UNICEF 2015, cuyo título fue "Reimaginar el Futuro" y el subtítulo, "innovación para todos los niños y niñas", hizo una apuesta atrevida y original, articulada en la incorporación y promoción de la innovación para amplificar las soluciones a los problemas que soportan la infancia más des-favorecida. Para dinamizar esta iniciativa lanzó la siguiente pregunta a los distintos sectores y ámbitos de intervención: “¿de qué manera pueden contribuir los innovadores a crear un mundo mejor?" y solicitaba explícitamente a las entidades y profesionales que publicaran sus propuestas y estrategias innovadoras en favor de la infancia.

En la tercera parte del informe, denominada "innovación para la equidad", definía el principio y a la vez objetivo que ha de regir todo tipo de innovación social: la equidad. Por tanto, la innovación necesaria es aquella que aumenta las oportunidades de aprovechar al máximo el potencial personal en el mayor número de niños posibles, o sea, todos. 
Al siguiente año, el informe anual sobre el Estado mundial de la infancia (UNICEF, 2016), titulado, "Una oportunidad para cada niño", y centrado en la Agenda 2030 para el Desarrollo Sostenible adoptada por la Asamblea General de la ONU, a favor de las personas, el planeta y la prosperidad, reconoce explícitamente la necesidad insoslayable de promover la equidad. En este informe se proclama a la Educación como uno de los motores de desarrollo más eficaces, a la vez que el mejor instrumento para lograr la equidad.

Con el término "equidad", UNICEF se refiere a que todas las personas en su infancia "tengan las mismas oportunidades de sobrevivir, crecer y alcanzar el pleno desarrollo de sus capacidades". Pero, según nos alerta el informe, una tercera parte del alumnado que cursa en nuestro mundo, la educación primaria, no tienen adquiridas las competencias básicas alfabéticas y numéricas. Alrededor del 38\% de los niños terminan la etapa de la educación primaria sin las competencias básicas de lectura, escritura y cálculo aritmético simple. Por lo que se defiende la necesidad de una educación de calidad que incremente los conocimientos, desarrolle la innovación, la prosperidad, y fomente sociedades incluyentes.

Los resultados de aprendizaje se relacionan con diversos tipos de ventajas y desventajas que provocan riesgo y vulnerabilidad y estos interactúan desde los primeros años de vida, por lo que el principio de equidad ha de asegurarse desde la educación infantil. En los "Objetivos de Desarrollo Sostenible" ya referidos anteriormente, se arenga a los gobiernos "a velar porque todas las niñas y todos los niños tengan acceso a servicios de atención y desarrollo en la primera infancia y a una enseñanza parvularia de calidad".

Estos servicios de atención y desarrollo han de ocuparse desde los primeros años de vida, tanto de la salud física como de la emocional de las personas.

\section{Vulnerabilidad y riesgo.}

En las interrelaciones del niño con los otros, el infante está recibiendo un tipo de educación informal. Recientes investigaciones desde la neurociencia, aportan datos relevantes sobre la influencia educativa de las experiencias vitales desde la primera infancia, tales como que las conversaciones en contextos significativos y funcionales para el niño, así como una alfabetización emergente y oportuna, correlacionan positivamente con el desarrollo de habilidades lingüísticas. Al contrario, la frecuente 
exposición a situaciones de estrés crónico, tales como por ejemplo, la privación nutricional y la violencia, pueden afectar a neuronas de zonas relacionadas con el aprendizaje y el desarrollo emocional. Las experiencias negativas vividas por el niño, pueden traducirse posteriormente tanto en dificultades en el aprendizaje, como en el desarrollo emocional y en la gestión de la ansiedad. Los servicios de atención y desarrollo, tanto físico como cognitivo y emocional, pueden coadyuvar en el desarrollo cerebral, fortaleciendo la capacidad de aprendizaje y fomentando la resistencia psicológica y la flexibilidad cognitivo emocional necesaria para adaptarse a los frecuentes cambios vitales que experimentan las personas (Center on the Developing Child of de Harvard University, 2016).

Al riesgo procedente de las desventajas socioeconómicas, puede añadírsele la baja o nula alfabetización de los progenitores. Los niños y niñas más aventajados socioeconómicamente, tienen cerca de 6 veces más probabilidades de adquirir las aptitudes básicas de alfabetización y aritmética que los de las familias en situación de pobreza cuyos progenitores, además, no fueron a la escuela (UNESCO, 2016).

Desde la Comisión Económica para América Latina y el Caribe (CEPAL), nos alertan de la transmisión intergeneracional de las oportunidades de bienestar y, especialmente, del grado de heredabilidad del nivel educativo. En el informe "La transformación productiva 20 años después: viejos problemas, nuevas oportunidades", esta comisión nos delata el hecho de que los jóvenes que tienen menos probabilidades de concluir la enseñanza secundaria son aquellos cuyos padres no terminaron la enseñanza formal (CEPAL, 2008). Además, no es cuestión exclusiva del acceso a la educación de calidad, sino también de la persistencia del alumnado en el sistema educativo hasta que logren los objetivos educativos (Marchesi \& Iglesias, 2008).

En la enseñanza secundaria, las pruebas internacionales de evaluación de estudiantes, muestran unos preocupantes niveles bajos de aprendizaje en muchos países de ingresos medianos y bajos. En estos estudios, el factor que más correlaciona con el riesgo de fracaso escolar es el nivel socioeconómico familiar. Ese condicionante se estimó en PISA mediante un índice de estatus social, cultural y económico (ESCS por sus siglas en inglés), configurado por el nivel educativo y de ocupación laboral de los progenitores, así como por un indicador de los materiales culturales en el hogar. En la prueba del Programa de Evaluación Internacional de Estudiantes PISA 2012 (OCDE, 2013), las diferencias en el índice ESCS explicaban, entre el alumnado de los países de 
la OCDE, alrededor del 15\% de la varianza observada en los resultados de matemáticas. En cuanto al fracaso escolar en la escolarización, uno de cada cinco estudiantes de los países de la OCDE, no termina la educación secundaria.

\section{Resiliencia}

En esta dirección, desde finales del siglo pasado, y con el objetivo de remediar y superar los efectos negativos en los aprendizajes y avances escolares asociados a la desventaja socieconómica, viene desarrollándose una línea de investigación exploratoria centrada en el conocimiento de los factores o variables que mejor correlacionan con ese pequeño porcentaje de alumnado que aún presentado ese riesgo, tiene éxito en sus estudios. A estos estudiantes se les considera «alumnado académicamente resiliente». (CoronadoHijón, 2017; Erberber, Stephens, Mamedova, Ferguson \& Kroeger, 2015).

Desde el campo de las Ciencias Sociales, los estudios sobre la resiliencia comenzaron desde el ámbito de la psicología infantil, a mitad de la década de los años 70 del siglo XX -con niños que vivían en contextos con desventaja sociocultural, con la finalidad de explicar cómo algunos niños y niñas eran capaces y competentes para superar adversidades y desarrollarse como personas saludables con perfiles de adaptación positiva. Desde este ámbito de la investigación básica se desplazó rápidamente al ámbito aplicado de la Educación Emocional, centrado en el aprendizaje de destrezas de resiliencia, entendido como un nuevo paradigma frente al abordaje de la vulnerabilidad y el riesgo, ya que- por el contrario- prioriza el enfoque en las fortalezas, en vez del déficit o dificultad. (Coronado-Hijón, 2016a).

Este nuevo ámbito de la Educación Emocional, cuyo término aparece por primera vez en el año 1966, en la revista Journal of Emotional Education, editada por el Institute of Applied Psychology de Nueva York, es un producto de la innovación educativa que ya ha cumplido la mayoría de edad y puede presentarse como postulante a este reto confluyente entre la innovación y la equidad para el objetivo perseguido de innovar en las soluciones a los problemas que soportan la infancia más desfavorecida.

La fundamentación de la comprensión científica de la influencia de las emociones en el pensamiento y el aprendizaje ha sido aportada por las investigaciones en neurociencia, las cuales han aportado datos que evidencian la correlación de las emociones con el aprendizaje, revelando que la emoción y la cognición son consecuentes con los procesos neuronales interdependientes, de tal forma que es 
literalmente imposible elaborar neurobiológicamente recuerdos sin la participación de las emociones (Immordino-Yang, 2016).

Desde que en 1993 la Organización Mundial de la Salud (OMS, 1997) lanzara su Iniciativa Internacional para la Educación en Habilidades para la Vida en las Escuelas (Life Skills Education in Schools) proponiendo su inclusión en la educación formal, éstas se han convertido en un campo prometedor de innovación educativa, con amplia proyección en distintas regiones del mundo.

El Life Skills Education in Schools es un modelo de intervención psicosocial que articula acciones de detección y prevención del riesgo; promueve estrategias de autocuidado y desarrolla competencias para la convivencia de los diferentes miembros y sectores de la comunidad escolar.

El propósito concreto de esta propuesta (OMS, 1997), es divulgar mundialmente desde la enseñanza formal, un grupo de diez destrezas genéricas psicosociales, seleccionadas por su consideración de máxima relevancia en la promoción de la competencia psicosocial de niñas, niños y jóvenes. La OMS, (1999) especificó que eran "aquellas aptitudes necesarias para tener un comportamiento adecuado y positivo, que nos permiten enfrentar eficazmente las exigencias y retos de la vida diaria", a saber: autoconocimiento, empatía, comunicación asertiva, relaciones interpersonales, toma de decisiones, manejo de problemas y conflictos, pensamiento creativo, pensamiento crítico, manejo de emociones y sentimientos y manejo de tensiones y estrés.

Paralelamente, otro organismo internacional, la Organización de las Naciones Unidas para la Educación, la Ciencia y la Cultura -en inglés- United Nations Educational, Scientific and Cultural Organization (UNESCO), encargó a una comisión internacional el estudio de la Educación para el siglo XXI, cuyo trabajo final quedó redactado en el informe denominado, «La Educación encierra un tesoro», (Delors, 1996). En éste se defiende la necesidad de ampliar los objetivos educativos, hasta entonces basados exclusivamente en contenidos cognitivos, a contenidos procedimentales y actitudinales o emocionales. Posteriormente en otro informe complementario, denominado, "Recomendación sobre las competencias clave para el aprendizaje permanente" (Comisión Europea, 2007), se destacaron una serie de aptitudes intervinientes en las ocho competencias clave, a saber: el pensamiento crítico, la creatividad, la capacidad de iniciativa, la resolución de problemas, la evaluación del 
riesgo, la toma de decisiones y la gestión constructiva de los sentimientos. Todas estas aptitudes, están implícitas en distinto grado, en la capacidad y respuesta resiliente.

En la revisión de estudios realizada el año 2003 por la investigadora Edith Henderson Grotberg, recogida en el libro "Resiliencia, descubriendo las propias fortalezas", en el capítulo denominado "Nuevas tendencias en resiliencia", destaca ocho evidencias relevantes de la investigación, así como los enfoques resultantes en torno al concepto de resiliencia, en esta área del desarrollo humano, a saber:

1. La resiliencia está ligada al desarrollo y el crecimiento humanos, con las consiguientes diferencias en edad.

2. La promoción de factores de resiliencia, así como la implementación y desarrollo de conductas resiliente necesitan diferentes estrategias formativas.

3. El nivel socioeconómico y la resiliencia no tienen una correlación siempre estable.

4. La resiliencia es diferente de los factores de riesgo y los factores de protección por sí solos. El producto no es igual a la suma de sus elementos.

5. La resiliencia puede ser medida como parte del bienestar y la calidad de vida de los sujetos.

6. Las diferencias culturales disminuyen cuando los sujetos se capacitan para valorar ideas nuevas y efectivas para su bienestar.

7. Prevención y promoción son conceptos clave en el desarrollo resiliente.

8. La resiliencia es un proceso que vertebra: factores, comportamientos y resultados resilientes.

Grotberg (2003) propone un modelo de promoción de la resiliencia, contextualizado en el ciclo vital de las personas y longitudinalmente en la dirección de las etapas del desarrollo descriptas por Erik Erikson: desarrollo de confianza básica (primer año de vida); desarrollo de autonomía (2 a 3 años de edad); iniciativa (de 4 a 6 años); sentido del logro y productividad (de 7 a 12 años) y finalmente; desarrollo de la identidad (de 13 a 19 años).

En otro trabajo, realizamos una revisión de los principales enfoques y programas en la educación de la resiliencia (Coronado-Hijón, 2016b) en el que se concluía que; de todas las modalidades de intervenciones posibles, es el enfoque ecosistémico holístico 
(Richardson, 2002) el que se ha mostrado más funcional. Éste ha de estar situado sistémicamente en el contexto de intervención.

\section{Conclusiones.}

Para alcanzar los objetivos de 2030, el informe anual sobre el Estado mundial de la infancia (UNICEF, 2016), titulado, "una oportunidad para cada niño", destaca la necesidad insoslayable de promover la equidad, proclamando a la educación como uno de los factores de desarrollo más eficaces, a la vez que se recomienda como el mejor instrumento para lograr la equidad. Los 17 objetivos y las 169 metas asociadas que las naciones del mundo se han comprometido a lograr son universales, ya que están vinculados a la promesa de "que nadie se quedara atrás... y nos esforzaremos por llegar primero a los más rezagados", Organización de las Naciones Unidas (2015).

Cuando UNICEF emplea el término "equidad" en el informe citado y otros documentos, se refiere a que todos los niños y niñas tengan las mismas oportunidades de sobrevivir, crecer y alcanzar un desarrollo pleno de sus capacidades.

Desde una educación de calidad, el informe asegura que, se puede terminar con los ciclos intergeneracionales de inequidad. La educación puede aportar a los niños y niñas, los conocimientos y las competencias necesarias para un saludable y adecuado nivel de vida. La educación de calidad correlaciona con un mayor nivel de ingresos económicos, una disminución de la pobreza y a una mejor salud.

Siguiendo la senda de innovación para todos los niños y niñas, propuesta por la UNICEF (2015), y apoyados en las últimas investigaciones de la neurociencia, es preciso avanzar tanto en el logro de una mejor salud física como emocional. Por lo que la educación debe ocuparse también de este ámbito tan relevante, tal como indican organismos internacionales como la Organización Mundial de la Salud (OMS, 1997) con su Iniciativa Internacional para la Educación en Habilidades para la Vida en las Escuelas (Life Skills Education in Schools) y la UNESCO, en el informe de la Educación para el siglo XXI, «La Educación encierra un tesoro», (Delors, 1996).

Uno de los mejores instrumentos para una educación emocional para la equidad, es el desarrollo y promoción de factores de resiliencia. 
Los ámbitos de intervención de la educación de la resiliencia son, fundamentalmente, el sociocomunitario y el escolar. En ambos la comunidad tiene un rol relevante desde un enfoque sistémico de la intervención.

Si en ambos ámbitos de intervención citados, el sector público tiene un rol preponderante, en el ámbito sociocomunitario, el tercer sector social, formado por las organizaciones de titularidad privada inscritas en un registro público con personalidad jurídica y que no tienen ánimo de lucro (reinvirtiendo sus beneficios en la propia actividad), con finalidades de promoción de la persona y para la inclusión de los colectivos vulnerables, pueden asumir un protagonismo incipiente en los próximos tiempos. Así pues, uno de los agentes claves en el tratamiento de las consecuencias de desventaja educativa, es el tercer sector social. La relación entre éstos y las familias constituye una alianza imprescindible en aras de: la reducción del absentismo escolar, el abandono temprano de la educación y la mejora del rendimiento.

Desde importantes estudios internacionales, se desprende que el perfil resultante de un centro eficaz en la generación de resiliencia académica en sujetos provenientes de entornos económicamente desfavorecidos (Erberber, Stephens, Mamedova, Ferguson \& Kroeger, 2015), es el que presenta un bajo número de alumnado en situación de pobreza, con una ratio por aula menor de veinte estudiantes en total, donde la educación emocional y las buenas prácticas de convivencia en el aula son un eje prioritario de la acción tutorial, y donde la participación del alumnado y las familias se aborda desde un planteamiento sistémico y colaborativo.

Los ámbitos citados funcionan como sistemas abiertos y en constante transformación, con relaciones interdependientes entre sus subsistemas (sujeto, familia, comunidad), de tal manera que un cambio en cualquiera de ellos puede elicitar cambios en todos los demás de manera holística y que, por tanto, es necesario atender a los procesos o secuencias interactivas que activan procesos de resiliencia desde un enfoque ecosistémico (Coronado-Hijón, 2010), tomando como unidad básica de intervención, al infante en su contexto.

Las estrategias de promoción y educación de la resiliencia no son totalmente generalizables, sino que, más bien, deben adaptarse según las circunstancias y particularidades de los estudiantes sobre las que los implementemos. En cualquier caso, todo proyecto de intervención debe atenerse a los siguientes criterios: 
a) Evaluación del contexto, en relación a las barreras que dificultan a los niños y sus familias, acceder a las oportunidades que necesitan para hacer realidad sus derechos de una educación de calidad y con equidad.

b) Diseñar soluciones accesibles para todos.

c) Evaluar las soluciones en cuanto a posibilidades y riesgos.

d) Adaptar y ampliar el espectro de soluciones.

e) Hacer partícipes a los infantes y sus familias, en el proceso.

Las líneas de innovación que proponemos han de pretender que las oportunidades equitativas para todos en el desarrollo emocional, social, cognitivo y del lenguaje, capaciten a todos para el éxito en la escuela y consecuentemente, en el trabajo y la comunidad.

Un factor de innovación educativa que puede coadyuvar de manera muy relevante en el éxito referido, es la resiliencia como paradigma de innovación en el desarrollo, ya que ofrece un marco teórico coherente fundamentado en los datos de investigación y en experiencias y buenas prácticas con evidencias de eficacia (Coronado-Hijón, 2016b).

Como conclusión final, unas magistrales frases de Boris Cyrulnik, (2002, p.215):

Cuando un niño sea expulsado de su hogar como consecuencia de un trastorno familiar, cuando se le coloque en una institución totalitaria, cuando la violencia del estado se extienda por todo el planeta, cuando los encargados de asistirle lo maltraten, cuando cada sufrimiento proceda de otro sufrimiento, como una catarata, será conveniente actuar sobre todas y cada una de las fases de la catástrofe: habrá un momento político para luchar contra esos crímenes, un momento filosófico para criticar las teorías que preparan esos crímenes, un momento técnico para reparar las heridas y un momento resiliente para retomar el curso de la existencia.

\section{Referencias bibliográficas}

Comisión Europea. (2007). Competencias clave para el aprendizaje permanente. Un marco de referencia europeo Luxemburgo: Oficina de Publicaciones Oficiales de las Comunidades Europeas. http://eurlex.europa.eu/LexUriServ/site/es/oj/2006/1_394/1_39420061230es0010 0018.pdf 
Coronado-Hijón, A. (2010). Orientación e intervención familiar en el contexto educativo desde el enfoque eco sistémico. Sevilla: Universidad Nacional de Educación a Distancia.

Coronado-Hijón, A. y Paneque, M. (2015). Resiliencia al fracaso escolar y desventaja sociocultural: un reto para la orientación y la tutoría. En Jiménez, S. y Silva, C. (Coord.) Trauma, contexto y exclusión: promocionando resiliencia. Granada: Grupo Editorial Universitario (GEU)

Coronado-Hijón, A. (2016a). Orientación y atención a la diversidad en las transiciones educativas interculturales. En, La Educación sí importa en el siglo XXI. Madrid: Editorial Síntesis, pp.257-269

Coronado-Hijón, A. (2016b). Educación de la Resiliencia Académica. En, Molero, M.M., Pérez-Fuentes, M.C., Gázquez, J.J., Barragán, A.B., Martos, A. y Simón, M.M. Variables Psicológicas y Educativas para la intervención en el ámbito escolar Vol II. Almería: ASUNIVEP

Coronado-Hijón, (2017). Academic resilience: a transcultural perspective. Procedia Social and Behavioral Sciences, 237

Center on the Developing Child (2015). The Science of Resilience (In-Brief). Harvard: Harvard University

Center on the Developing Child of de Harvard University 'Brain Architecure. Harvard: Harvard University. <http://developingchild.harvard.edu/science/keyconcepts/brain-architecture>, consultado el 1 de marzo de 2016.

CEPAL (Comisión Económica para América Latina y el Caribe) (2008). La transformación productiva 20 años después: viejos problemas, nuevas oportunidades. (LC/G.2367 (SES.32/3) P). NU. CEPAL

Cyrulnik, B. (2002). Los patitos feos. Barcelona: Gedisa.

Cyrulnik, B. (2015). Las almas heridas. Barcelona: Gedisa.

Delors, J., (1996). La educación encierra un tesoro. Informe a la UNESCO de la Comisión internacional sobre la educación para el siglo XXI, Madrid, España: Santillana/UNESCO 
Erberber, E., Stephens, M., Mamedova, S., Ferguson, S. \& Kroeger, T. (2015). Alumnos socioeconómicamente desfavorecidos que tienen éxito académico: Examen transnacional de la resiliencia académica. IEA, Policy Brief Series, $\mathrm{n}^{\mathrm{o}} 5$, Amsterdam, IEA, http://www. iea.nl/policy_briefs.html

Grotberg, E., (2003). Resiliencia, descubriendo las propias fortalezas. Barcelona: Paidós.

Immordino-Yang, M.H., (2016). Las Emociones, el Aprendizaje y el cerebro. WW Norton \& Co

Marchesi, Á., \& Iglesias, E. V. (2008). Metas educativas 2021: la educación que queremos para la generación de los bicentenarios. Transatlántica de Educación, (5), 113-129.

OECD (2013). PISA 2012 Results: What Students Know and Can Do. 4 vols. Paris: OECD.

Organización de las Naciones Unidas, (2015) Transformar nuestro mundo: la Agenda 2030 para el Desarrollo Sostenible, Resolucion de la Asamblea General A/RES/70/1, parrafo 4. Nueva York: Naciones Unidas.

Organización Mundial de la Salud, (OMS), (1997). Life Skills Education in Schools. Ginebra, Suiza.

Organización Mundial de la Salud, (OMS), (1999). Guidelines: Life Skills Education Curricula for Schools. Ginebra, Suiza,

Richardson, G. E. (2002). The meta-theory of resilience and resiliency. Journal of Clinical Psychology, 58, 307-321. http://dx.doi.org/10.1002/jclp.10020

Torres, V. L. (2010). Educación y resiliencia: alas de la transformación so-cial. Revista Electrónica. Actualidades Investigativas en Educación, 10(2), 1-14.

UNICEF (2015). The State of the World's Children 2015: Reimagine the future. Nueva York: UNICEF.

UNICEF (2016). The State of the World's Children 2016: A fair chance for every child. Nueva York: UNICEF. 